\title{
Cycloartanes from Oxyanthus pallidus and derivatives with analgesic activities
}

\author{
Basile Nganmegne Piegang ${ }^{1}$, Ignas Bertrand Nzedong Tigoufack², David Ngnokam², Angèle Sorel Achounna',
} Pierre Watcho', Wolfgang Greffrath ${ }^{3}$, Rolf-Detlef Treede ${ }^{3}$ and Télesphore Benoît Nguelefack ${ }^{1 *}$

\begin{abstract}
Background: The leaves of Oxyanthus pallidus Hiern (Rubiaceae) are extensively used in the west region of Cameroon as analgesic. These leaves are rich in cycloartanes, a subclass of triterpenes known to possess analgesic and anti-inflammatory properties. The present study aimed at evaluating the analgesic properties of three cycloartanes isolated from Oxyanthus pallidus leaves as well as their aglycones and acetylated derivatives.

Methods: Three cycloartanes $\mathrm{OP}_{3}, \mathrm{OP}_{5}$ and $\mathrm{OP}_{6}$ obtained by successive chromatography of the crude methanol extract of the leaves were hydrolysed to yield respective aglycone $A O P_{1}, A_{O P}, A_{2} P_{3}$ and acetylated to $\mathrm{HOP}_{1}, \mathrm{HOP}_{2}$ and $\mathrm{HOP}_{3}$ respectively. Formalin-induced pain model was used to evaluate the acute anti-nociceptive properties of these cycloartanes $(5 \mathrm{mg} / \mathrm{kg}, \mathrm{p.o})$ in mice and to determine the structure-activity relationship. Acute $(24 \mathrm{~h})$ and chronic (10 days) anti-hyperalgesic and anti-inflammatory activities of $\mathrm{OP}_{5}$ were evaluated at the doses of 2.5 and $5 \mathrm{mg} / \mathrm{kg} /$ day administered orally. $\mathrm{OP}_{6}$ was also evaluated in acute experiments. The antioxidant and hepatoprotective activities of $\mathrm{OP}_{5}$ were evaluated at the end of the chronic treatment.

Results: The mixture and the individual isolated cycloartanes significantly inhibited both phases of formalininduced pain with percentage inhibition ranging from 13 to $78 \%$. Acid hydrolysis did not significantly affect their antinociceptive activities while acetylation significantly reduced the effects of these compounds during the second phase of pain. $\mathrm{OP}_{5}$ and $\mathrm{OP}_{6}$ induced acute anti-hyperalgesic activity in formalin-induced mechanical hyperalgesia but not an anti-inflammatory effect. Repeated administration of $\mathrm{OP}_{5}$ for 10 days did not induce any antihyperalgesic effect. The evaluation of in vivo antioxidant properties showed that $\mathrm{OP}_{5}$ significantly reduced malondialdehyde and increased superoxide dismutase levels in liver without significantly affecting other oxidative stress and hepatotoxic parameters. Chronic administration of $\mathrm{OP}_{5}$ did not cause gastric ulceration.

Conclusion: Cycloartanes isolated from Oxyanthus pallidus possess analgesic effects but lack anti-inflammatory activities. This analgesic effect especially on inflammatory pain may be due to the presence of hydroxyl group in front of the plane. $\mathrm{OP}_{5}$ is devoid of ulcerogenic effect and possess antioxidant properties that might be of benefit to its analgesic properties.
\end{abstract}

Keywords: Pain, Inflammation, Oxyanthus pallidus, Cycloartanes, Structure-activity relationship

\footnotetext{
* Correspondence: nguelefack@yahoo.fr

${ }^{1}$ Laboratory of Animal Physiology and Phytopharmacology, Department of

Animal Biology, Faculty of Science, University of Dschang, P.O. Box 67,

Dschang, Cameroon

Full list of author information is available at the end of the article
} 


\section{Background}

Pain is known as a major public health threat not only because it underlies almost every illness but also because it has become an illness by himself. It is therefore one of the leading causes of medical consultation [1], with enormous socio-economic and physiological deleterious impacts. Therefore, there is a high need to properly handle this ailment especially at its chronic stage. Huge progress has been made in recent years in order to first understand the pathology and secondly to master the therapeutic aspects and treatment. For the last purpose, a large number of therapeutics molecules like opioids, non-steroidal and steroidal analgesics have been developed. But existing therapeutics have some disadvantages such as their inefficacy, the resistance of some pathologies and the side effects, especially gastrointestinal, hepatic or renal toxicity. For instance, patients with severe inflammatory diseases respond poorly to conventional doses of corticosteroids $[2,3]$. One of the exciting strategies to overcome these problems is to search for new and better drugs with minimal adverse effects. An important field of investigation to discover new therapeutic molecules that can treat more efficiently pain is that of secondary metabolites present in medicinal plants. In fact phytopharmacological research has brought substantial contribution to drug innovation. In addition, chemical structural modification of molecules has been shown to strongly modify the biological activity of initial compounds. In fact, the analgesic activity of a good number of chemical has been improved by their structural modification [4-6].

Triterpenes constitute a class of secondary metabolites that have shown potent analgesic and anti-inflammatory activities [7-9], one of the subclass being cycloartanes. The lasts molecules are secondary metabolites belonging to the class of tetracyclic triterpenes and are synthesized by many plant species. Cycloartanes possess various biological activities including anti-tumour, anti-virus, antibacterial, anti-inflammatory, analgesic or antioxidant activities $[10,11]$. They therefore, offer an alternative in the treatment of pain.

Ayatollahi et al. [12] showed that cycloartanes are able to deactivate protein kinase $\mathrm{C}$ (PKC) and that this effect could be attributed to the presence of hydroxyl group in their molecular structures. Knowing the pivotal role that PKC plays in the transduction of pain $[13,14]$, we hypothesized that cycloartanes may possess good analgesic activity related to the presence of hydroxyl group.

The present study evaluates the analgesic and antiinflammatory activities of three cycloartane glycosides, named pallidioside $\mathrm{A}\left(\mathrm{OP}_{3}\right)$, pallidioside $\mathrm{B}\left(\mathrm{OP}_{5}\right)$ and pallidioside $\mathrm{C}\left(\mathrm{OP}_{6}\right)$ isolated from the leaves of Oxyanthus pallidus, a plant extensively used in the West region of Cameroon as analgesic. In attempt to determine the importance of the glycoside moiety and the hydroxyl group in the biological activity of these compounds, the effects of their respective aglycone $\left(\mathrm{AOP}_{1}, \mathrm{AOP}_{2}, \mathrm{AOP}_{3}\right)$ and acetylated $\left(\mathrm{HOP}_{1}, \mathrm{HOP}_{2}, \mathrm{HOP}_{3}\right)$ derivatives were also evaluated.

\section{Methods \\ Animal}

Male and female rats of the Wistar strain aged 3 months and weighing between 180 and $200 \mathrm{~g}$ and Swiss mice of both sexes, aged 3 months and weighing between 20 and $30 \mathrm{~g}$ were used in this study. These animals were bred in the animal house of the Laboratory of Animal Physiology and Phytopharmacology of the University of Dschang under a 12-h light/dark cycle with free access to standard commercialized rodent diet and water. The number of animals used was the minimum possible to determine consistent effects of the drug treatments (See figures). All protocols were submitted and approved by the local Ethics Committee "The Animal Ethics Committee of the University of Dschang" and conformed to the guidelines for the study of pain in awake animals established by the International Association for the Study of Pain.

\section{Plant collection and extraction Collection and isolation of major compounds}

Oxyanthus pallidus materials were harvested in November in Dschang in the Western region of Cameroon. The plant was identified at the National Herbarium of Cameroon in Yaounde by Mr. Victor Nana, in comparison to an existing Voucher specimen $n^{\circ} 7335 /$ SFR/CAM. The leaves were subsequently dried at room temperature and then crushed. The resulting powder was extracted by soaking in methanol for $24 \mathrm{~h}$. After filtration and evaporation at $70{ }^{\circ} \mathrm{C}$ under reduced pressure, the extract obtained was subsequently subjected to repeated column chromatography silica gel, which allowed to isolate and identify the three main compounds pallidiosides $\mathrm{A}\left(\mathrm{OP}_{3}\right)$, pallidiosides $\mathrm{B}\left(\mathrm{OP}_{5}\right)$ and pallidiosides $\mathrm{C}\left(\mathrm{OP}_{6}\right)$ as previously described [15].

\section{Preparation of aglycone derivatives}

One point five gram of each compound $\left(\mathrm{OP}_{3}, \mathrm{OP}_{5}\right.$ and $\mathrm{OP}_{6}$ ) was individually refluxed in methanol $/ \mathrm{H}_{2} \mathrm{SO}_{4} 10 \%$ $(25 \mathrm{ml})$ at room temperature for $48 \mathrm{~h}$. Reaction mixture was neutralized by sodium hydroxide solution $(6 \mathrm{~N})$ and extracted with $\mathrm{CHCl}_{3}(3 \times 10 \mathrm{ml})$. Each organic phase was concentrated under vacuum to give residues (560, 530 and $540 \mathrm{mg}$ respectively) which were separately subjected to $\mathrm{CC}$ over silica gel with hexane/AcOEt (1:1) as the eluent to yield $\mathrm{AOP}_{1}, \mathrm{AOP}_{2}$ and $\mathrm{AOP}_{3}$ compounds (350, 335 and $340 \mathrm{mg}$ respectively).

\section{Preparation of acetylated derivatives}

Two hundred and fifty milligrams of each compound $\left(\mathrm{OP}_{3}, \mathrm{OP}_{5}\right.$ and $\left.\mathrm{OP}_{6}\right)$ were individually acetylated with $\mathrm{Ac}_{2} \mathrm{O}$-pyridine $(2: 1) \mathrm{v} / \mathrm{v}$ at room temperature for $24 \mathrm{~h}$, 


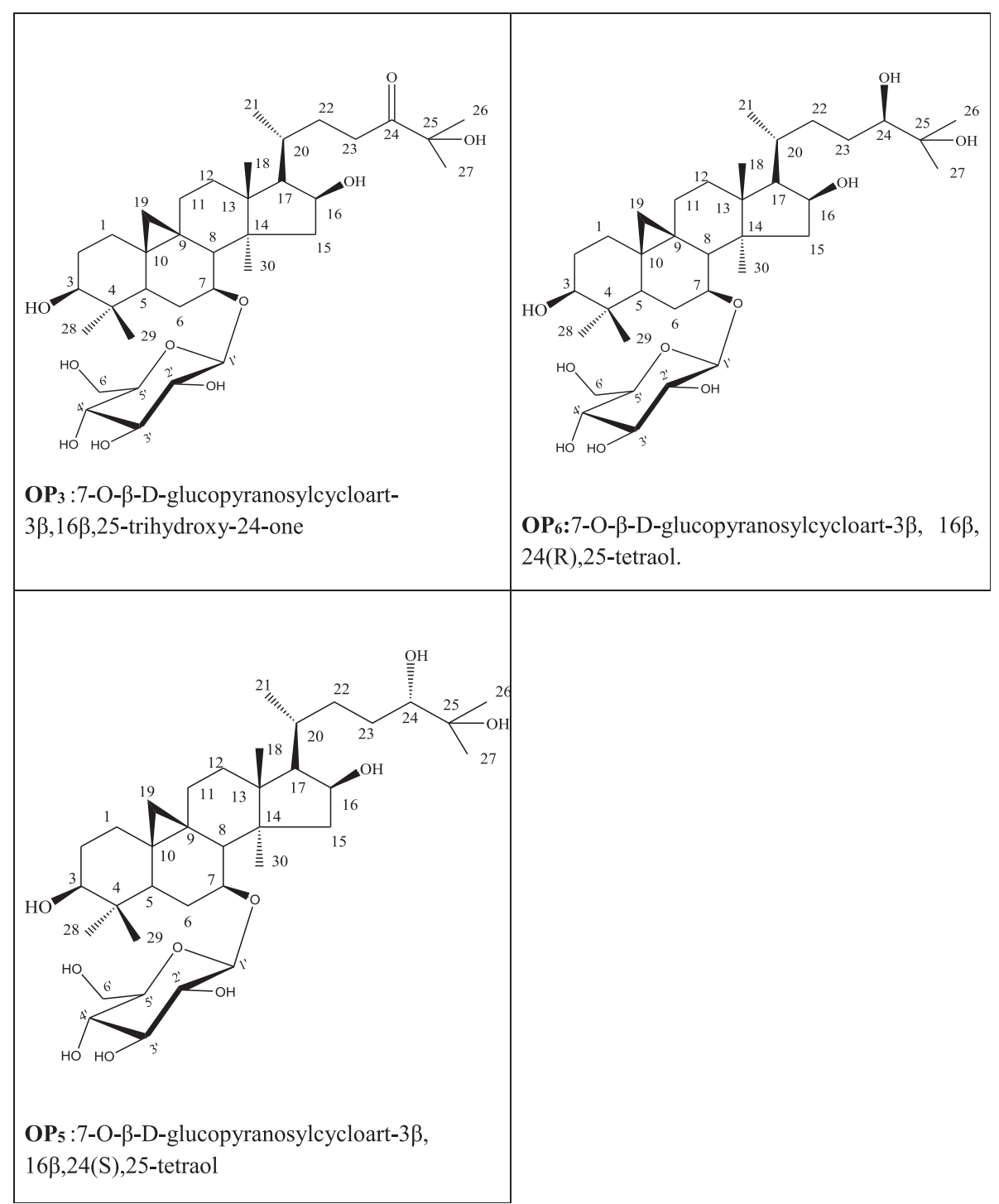

Fig. 1 Structures of the main cycloartanes (pallidioside $A: O P_{3}$, pallidioside $B: O_{5}$ and pallidioside $C: P_{6}$ ) isolates from the leaves of $O x y a n t h u s ~ p a l l i d u s$

and each of the reaction mixture was purified over silica gel column, eluted with the hex-EtOAc (50 \%) mixture to give the corresponding acetylated derivatives $\mathrm{HOP}_{1}$, $\mathrm{HOP}_{2}$ and $\mathrm{HOP}_{3}$ (270, 280 and 285 mg respectively).

\section{Pharmacological tests}

\section{Spontaneous pain induced by formalin in mice}

This experiment followed the procedure previously described [16]. Animals were orally treated (gavage) with the mixture of the three cycloartanes $(5,10$ and $20 \mathrm{mg} / \mathrm{kg})$, individual isolated compounds $(5 \mathrm{mg} / \mathrm{kg}$ ) or derivatives ( $5 \mathrm{mg} / \mathrm{kg}$ ). Diclofenac given orally at $5 \mathrm{mg} / \mathrm{kg}$ was used as positive control while the negative control group received $0.9 \% \mathrm{NaCl}$ solution. All the oral administered substances were given at equivalent volume of $0.5 \mathrm{ml} / 30 \mathrm{~g}$ body weight. One hour after administration of different substances, pain was induced by injecting $20 \mu \mathrm{L}$ of formalin ( $2.5 \%$ in saline) under the aponeurosis of the dorsal surface of the right hind paw of each animal. They were individually observed and pain evidenced by licking, flinching or biting the injected paw was quantified using an electronic timer, in two periods. The first period consisted of the first $5 \mathrm{~min}$ indicating neurogenic pain and second period (15th to 45 th minute) corresponding to the tonic inflammatory pain.

\section{Mechanical hyperalgesia and inflammation induced by formalin in rats}

In an attempt to evaluate the time-dependent analgesic and anti-inflammatory effects, $\mathrm{OP}_{5}$ and $\mathrm{OP}_{6}$ were tested 


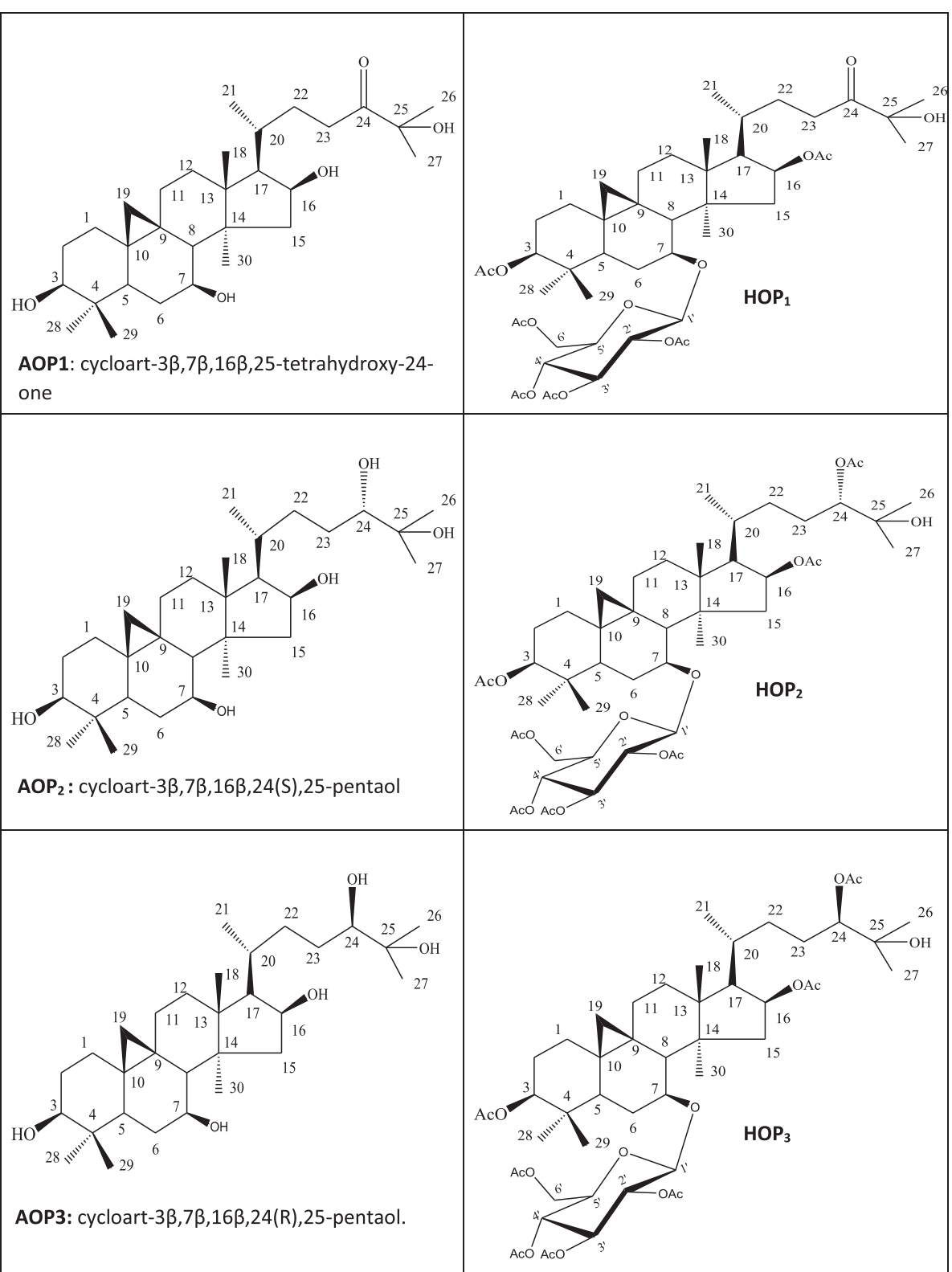

Fig. 2 Structures of respective aglycones $\left(A O P_{1}, A O P_{2}, A O P_{3}\right)$ and acetylated $\left(H O P_{1}, H O P_{2}\right.$ and $\left.H O P_{3}\right)$ derivatives of $O P_{3}, O P_{5}$ and $O P_{6}$

acutely in formalin-induced pain. The effect of 10 days repeated administration of $\mathrm{OP}_{5}(2.5$ and $5 \mathrm{mg} / \mathrm{kg} /$ day as a single bolus) was also tested. $\mathrm{NaCl} 0.9 \%$ and diclofenac were respectively used for negative and positive controls. One hour after oral administration of various substances, pain and inflammation were induced by injecting $0.1 \mathrm{ml}$ of a $2 \%$ formalin solution, under the left hind paw of each rat [17]. Ugo Basile analgesy meter no. 372157 and the caliper were used to measure the response threshold of each animal to mechanical pain and the paw diameter, respectively. The response threshold of the animal to mechanical pain was reported before treatment and 1, 2, 4, 6, 8 and $24 \mathrm{~h}$ post injection for the first day and once a day for ten days. The diameter of the injected paw was measured before treatment and 3, 5, 7 and 24 h post injection for the first day and then once a day for ten days. For repeated treatments, drugs were administered once a day, after collection of data. At the end of the experiment, the antioxidant, hepatotoxic and ulcerogenic effects of chronic administration of $\mathrm{OP}_{5}$ were examined. On this purpose, animals were sacrificed on day 11 . Blood, kidney and liver samples were collected. Serum was separated for ALAT and ASAT assays. Kidney and liver were homogenized in Tris buffer and centrifuged. The supernatant was used to assay nitric oxide, malondialdehyde, superoxide dismutase, catalase and glutathione. 
Table $1{ }^{1} \mathrm{H}(600 \mathrm{MHz}, \mathrm{MeOD})$ and ${ }^{13} \mathrm{C}(150 \mathrm{MHz}, \mathrm{MeOD})$ data of $\mathrm{AOP}_{1}, \mathrm{AOP}_{2}$ and $\mathrm{AOP}_{3}$

\begin{tabular}{|c|c|c|c|c|c|c|}
\hline \multirow[t]{2}{*}{ Positions } & \multicolumn{2}{|l|}{$\mathrm{AOP}_{1}$} & \multicolumn{2}{|c|}{$\mathrm{AOP}_{2}$} & \multicolumn{2}{|c|}{$\mathrm{AOP}_{3}$} \\
\hline & $\delta_{C}$ & $\delta_{H}(m u l t, J(H z)$ & $\overline{\delta_{C}}$ & $\delta_{H}(m u l t, J(H z)$ & $\delta_{C}$ & $\delta_{H}(m u l t, J(H z)$ \\
\hline 1 & 31.5 & $1.33(1 \mathrm{H}, \mathrm{m}, \mathrm{H \beta}), 1.61(1 \mathrm{H}, \mathrm{m}, \mathrm{Ha})$ & 31.3 & $1.33(1 \mathrm{H}, \mathrm{m}, \mathrm{H \beta}), 1.60(1 \mathrm{H}, \mathrm{m}, \mathrm{Ha})$ & 31.3 & $1.33(1 \mathrm{H}, \mathrm{m}, \mathrm{H} \beta), 1.62(1 \mathrm{H}, \mathrm{m}, \mathrm{Ha})$ \\
\hline 2 & 30.5 & $1.64(1 \mathrm{H}, \mathrm{m}, \mathrm{Ha}), 1.73(1 \mathrm{H}, \mathrm{m}, \mathrm{H} \beta)$ & 29.4 & $1.64(1 \mathrm{H}, \mathrm{m}, \mathrm{Ha}), 1.73(1 \mathrm{H}, \mathrm{m}, \mathrm{H \beta})$ & 29.4 & $1.63(1 \mathrm{H}, \mathrm{m}, \mathrm{Ha}), 1.72(1 \mathrm{H}, \mathrm{m}, \mathrm{H} \beta)$ \\
\hline 3 & 79.1 & $3.17(\mathrm{dd}, 4.6,10.1)$ & 77.7 & $3.24(\mathrm{dd}, 4.4,11.1)$ & 78.0 & $3.17(\mathrm{dd}, 4.5,10.1)$ \\
\hline 4 & 41.2 & & 39.8 & / & 39.8 & / \\
\hline 5 & 45.6 & $1.51(\mathrm{dd}, 3.7,13.1)$ & 45.7 & $1.52(\mathrm{dd}, 3.9,13.1)$ & 45.7 & $1.51(\mathrm{dd}, 3.8,12.1)$ \\
\hline 6 & 29.3 & $1.04(1 \mathrm{H}, \mathrm{m}, \mathrm{Ha}), 1.74(1 \mathrm{H}, \mathrm{m}, \mathrm{H} \beta)$ & 30.7 & $1.04(1 \mathrm{H}, \mathrm{m}, \mathrm{Ha}), 1.74(1 \mathrm{H}, \mathrm{m}, \mathrm{H \beta})$ & 30.7 & 1.03, $(1 \mathrm{H}, \mathrm{m}, \mathrm{Ha}), 1.74(1 \mathrm{H}, \mathrm{m}, \mathrm{H \beta})$ \\
\hline 7 & 70.1 & 3.50 ddd $(4,0,7.1,12.6)$ & 70.0 & $3.50 \mathrm{ddd}(4,0,7.1,12.7)$ & 70.0 & $3.50(\mathrm{ddd}, 4.0,9.4,13.3)$ \\
\hline 8 & 54.4 & $1.72(1 \mathrm{H}, \mathrm{d}, 7.0)$ & 54.2 & $1.71(1 \mathrm{H}, \mathrm{d}, 6.0)$ & 54.2 & $1.72(1 \mathrm{H}, \mathrm{d}, 6.0)$ \\
\hline 9 & 26.6 & & 26.5 & / & 26.5 & / \\
\hline 10 & 19.7 & & 19.6 & / & 19.7 & / \\
\hline 11 & 26.0 & $1.31(1 \mathrm{H}, \mathrm{m}, \mathrm{Ha}), 1.90(1 \mathrm{H}, \mathrm{m}, \mathrm{H} \beta)$ & 26.0 & $1.31(1 \mathrm{H}, \mathrm{m}, \mathrm{Ha}), 1.92(1 \mathrm{H}, \mathrm{m}, \mathrm{H \beta})$ & 26.0 & $1.30(1 \mathrm{H}, \mathrm{m}, \mathrm{Ha}), 1.92(1 \mathrm{H}, \mathrm{m}, \mathrm{H} \beta)$ \\
\hline 12 & 32.8 & $1.61(1 \mathrm{H}, \mathrm{m}, \mathrm{Ha}), 1.68(1 \mathrm{H}, \mathrm{m}, \mathrm{H \beta})$ & 32.4 & $1.63(1 \mathrm{H}, \mathrm{m}, \mathrm{Ha}), 1.69(1 \mathrm{H}, \mathrm{m}, \mathrm{H \beta})$ & 32.3 & $1.62(1 \mathrm{H}, \mathrm{m}, \mathrm{Ha}), 1.68(1 \mathrm{H}, \mathrm{m}, \mathrm{H} \beta)$ \\
\hline 13 & 45.3 & & 45.4 & / & 45.4 & / \\
\hline 14 & 46.0 & & 46.0 & / & 46.0 & / \\
\hline 15 & 48.8 & $\begin{array}{l}1.64(1 \mathrm{H}, \mathrm{dd}, 5.2,7.5, \mathrm{H} \beta), 2.25 \\
(1 \mathrm{H}, \mathrm{dd}, 5.27 .5, \mathrm{Ha})\end{array}$ & 48.8 & $\begin{array}{l}1.66(1 \mathrm{H}, \mathrm{dd}, 5.2,7.6, \mathrm{HB}), 2.25 \\
(1 \mathrm{H}, \mathrm{dd}, 5.2,7.6, \mathrm{Ha})\end{array}$ & 48.8 & $\begin{array}{l}1.67(1 \mathrm{H}, \mathrm{dd}, 5.2,7.7, \mathrm{HB}), 2.25 \\
(1 \mathrm{H}, \mathrm{dd}, 5.2,7.7, \mathrm{Ha})\end{array}$ \\
\hline 16 & 72.1 & $4.43(1 \mathrm{H}, \mathrm{ddd}, 5.2,7.5,7.8)$ & 72.1 & $4.44(1 \mathrm{H}, \mathrm{ddd}, 5.2,7.6,7.9)$ & 72.0 & $4.43(1 \mathrm{H}, \mathrm{ddd}, 5.2,7.6,7.9)$ \\
\hline 17 & 55.8 & $1.63(1 \mathrm{H}, \mathrm{dd}, 7.8,12.3)$ & 55.9 & $1.64(1 \mathrm{H}, \mathrm{dd}, 7.9,12.4)$ & 55.8 & $1.63(1 \mathrm{H}, \mathrm{dd}, 7.9,12.4)$ \\
\hline 18 & 17.2 & $1.20(3 \mathrm{H}, \mathrm{s})$ & 17.3 & $1.22(3 \mathrm{H}, \mathrm{s})$ & 17.2 & $1.21(3 \mathrm{H}, \mathrm{s})$ \\
\hline 19 & 27.9 & $0.19(1 \mathrm{H}, \mathrm{d}, 4.0, \mathrm{Ha}), 0.87(1 \mathrm{H}, \mathrm{d}, 4.0, \mathrm{H} \beta)$ & 27.9 & $\begin{array}{l}0.19(1 \mathrm{H}, \mathrm{d}, 4.0, \mathrm{Ha}), 0.85 \\
(1 \mathrm{H}, \mathrm{d}, 4.0, H \beta)\end{array}$ & 27.9 & $\begin{array}{l}0.75(1 \mathrm{H}, \mathrm{d}, 4.0, \mathrm{Ha}), 0.86 \\
(1 \mathrm{H}, \mathrm{d}, 4.0, \mathrm{H} \beta)\end{array}$ \\
\hline 20 & 30.1 & $1.81(1 \mathrm{H}, \mathrm{m})$ & 28.4 & $1.92(1 \mathrm{H}, \mathrm{m})$ & 28.5 & $1.82(1 \mathrm{H}, \mathrm{m})$ \\
\hline 21 & 17.5 & $0.99(1 \mathrm{H}, \mathrm{d}, 7.6)$ & 17.6 & $0.99(1 \mathrm{H}, \mathrm{d}, 7.6)$ & 17.5 & $1.00(1 \mathrm{H}, \mathrm{d}, 7.6)$ \\
\hline 22 & 31.1 & $1.26(1 \mathrm{H}, \mathrm{m}, \mathrm{Ha})_{,} 1.82(1 \mathrm{H}, \mathrm{m}, \mathrm{H \beta})$ & 32.3 & $\begin{array}{l}1.24(1 \mathrm{H}, \mathrm{m}, \mathrm{Ha}), 1.82 \\
(1 \mathrm{H}, \mathrm{m}, \mathrm{H \beta})\end{array}$ & 32.5 & $1.22(1 \mathrm{H}, \mathrm{m}, \mathrm{Ha})_{1} 1.92(1 \mathrm{H}, \mathrm{m}, \mathrm{H \beta})$ \\
\hline 23 & 28.3 & $1.63(1 \mathrm{H}, \mathrm{m}, \mathrm{Ha}), 1.70(1 \mathrm{H}, \mathrm{m}, \mathrm{H} \beta)$ & 27.0 & $\begin{array}{l}1.63(1 \mathrm{H}, \mathrm{m}, \mathrm{Ha}), 1.72 \\
(1 \mathrm{H}, \mathrm{m}, \mathrm{H \beta})\end{array}$ & 28.0 & $1.62(1 \mathrm{H}, \mathrm{m}, \mathrm{Ha}), 1.72(1 \mathrm{H}, \mathrm{m}, \mathrm{H} \beta)$ \\
\hline 24 & 218.9 & & 77.0 & $3.40(1 \mathrm{H}, \mathrm{dd}, 5.1,12.0)$ & 79.3 & $3.25(1 \mathrm{H}, \mathrm{dd}, 4.9,11 . .3)$ \\
\hline 25 & 77.8 & & 72.4 & / & 72.4 & / \\
\hline 26 & 26.8 & $1.19(3 \mathrm{H}, \mathrm{s})$ & 24.0 & $1.19(3 \mathrm{H}, \mathrm{s})$ & 24.3 & $1.19(3 \mathrm{H}, \mathrm{s})$ \\
\hline 27 & 26.8 & $1.17(3 \mathrm{H}, \mathrm{s})$ & 23.9 & $1.17(3 \mathrm{H}, \mathrm{s})$ & 23.4 & $1.16(3 \mathrm{H}, \mathrm{s})$ \\
\hline 28 & 26.0 & $0.98(3 \mathrm{H}, \mathrm{s})$ & 24.6 & $0.98(3 \mathrm{H}, \mathrm{s})$ & 24.6 & $0.98(3 \mathrm{H}, \mathrm{s})$ \\
\hline 29 & 13.2 & $0.86(3 \mathrm{H}, \mathrm{s})$ & 13.1 & $0.84(3 \mathrm{H}, \mathrm{s})$ & 14.9 & $0.86(3 \mathrm{H}, \mathrm{s})$ \\
\hline 30 & 18.5 & $0.97(3 \mathrm{H}, \mathrm{s})$ & 18.4 & $0.97(3 \mathrm{H}, \mathrm{s})$ & 18.4 & $0.97(3 \mathrm{H}, \mathrm{s})$ \\
\hline
\end{tabular}

Immediately after blood, liver and kidney collection, the stomach was carefully dissected out and opens along the great curvature. The mucus weight and ulceration index were estimated as previously described [18].

\section{Biochemical analysis}

To assess whether different treatments could induce liver damage, enzymatic activities of aspartate aminotrasferase (AST) and alanine aminotrasferase (ALT) were measured in serum following the commercial kit (Immesco) procedure. SOD was evaluated based on its ability to inhibit or retard the autoxidation of adrenaline to adrenochrome in basic medium [19]. To assess whether the tested compound could inhibit lipid peroxidation, the amount of malondialdehyde was assayed in homogenates of liver and kidney. The protocol followed was as previously described by Ohkawa et al. [20]. Glutathione concentration and catalase activity in tissues homogenates were measured according to the methods described by Dimo et al. [21]. NO content in the homogenates of kidney and liver was measured, using Fiddler's method [22]. 
Table $2{ }^{1} \mathrm{H}(500 \mathrm{MHz}, \mathrm{MeOD})$ and ${ }^{13} \mathrm{C}(150 \mathrm{MHz}, \mathrm{MeOD})$ data of $\mathrm{HOP}_{1}, \mathrm{HOP}_{2}$ and $\mathrm{HOP}_{3}$

\begin{tabular}{|c|c|c|c|c|c|c|}
\hline \multirow[t]{2}{*}{ Positions } & \multicolumn{2}{|l|}{$\mathrm{HOP}_{1}$} & \multicolumn{2}{|l|}{$\mathrm{HOP}_{2}$} & \multicolumn{2}{|l|}{$\mathrm{HOP}_{3}$} \\
\hline & $\delta_{C}$ & $\delta_{H}(m u l t, J(H z)$ & $\delta_{C}$ & $\delta_{\mathrm{H}}($ mult, $J(\mathrm{~Hz})$ & $\delta_{C}$ & $\delta_{H}(m u l t, J(H z)$ \\
\hline 1 & 30.4 & $1.40(1 \mathrm{H}, \mathrm{m}, \mathrm{H} \beta), 1.60(1 \mathrm{H}, \mathrm{m}, \mathrm{Ha})$ & 29.1 & $1.62(1 \mathrm{H}, \mathrm{m}, \mathrm{H} \beta), 1.72(1 \mathrm{H}, \mathrm{m}, \mathrm{Ha})$ & 29.1 & $1.62(1 \mathrm{H}, \mathrm{m}, \mathrm{H} \beta), 1.72(1 \mathrm{H}, \mathrm{m}, \mathrm{Ha})$ \\
\hline 2 & 29.1 & $1.62(1 \mathrm{H}, \mathrm{m}, \mathrm{Ha}), 1.73(1 \mathrm{H}, \mathrm{m}, \mathrm{H} \beta)$ & 27.0 & $1.43(1 \mathrm{H}, \mathrm{m}, \mathrm{Ha}), 1.62(1 \mathrm{H}, \mathrm{m}, \mathrm{H} \beta)$ & 27.0 & $1.43(1 \mathrm{H}, \mathrm{m}, \mathrm{Ha}), 1.62(1 \mathrm{H}, \mathrm{m}, \mathrm{H} \beta)$ \\
\hline 3 & 77.8 & $3.22(\mathrm{dd}, 4.4,11.1)$ & 77.8 & $3.23(\mathrm{dd}, 4.2,10.9)$ & 77.8 & $3.23(\mathrm{dd}, 4.2,10.9)$ \\
\hline 4 & 39.8 & / & 39.8 & / & 39.8 & / \\
\hline 5 & 44.3 & $1.42(\mathrm{dd}, 3.9,13.3)$ & 44.2 & $1.45(\mathrm{dd}, 3.9,13.1)$ & 44.2 & $1.45(\mathrm{dd}, 3.9,13.1)$ \\
\hline 6 & 28.1 & $1.08(1 \mathrm{H}, \mathrm{m}, \mathrm{Ha}), 2.13(1 \mathrm{H}, \mathrm{m}, \mathrm{H} \beta)$ & 28.1 & $1.10(1 \mathrm{H}, \mathrm{m}, \mathrm{Ha}), 2.13(1 \mathrm{H}, \mathrm{m}, \mathrm{H} \beta)$ & 28.1 & $1.10(1 \mathrm{H}, \mathrm{m}, \mathrm{Ha}), 2.13(1 \mathrm{H}, \mathrm{m}, \mathrm{H} \beta)$ \\
\hline 7 & 80.0 & $3.63(\mathrm{ddd}(4,0,7.63,12.6)$ & 80.0 & $3.63(\mathrm{ddd}(4,0,7.1,12.7)$ & 80.0 & $3.63(\mathrm{ddd}(4,0,7.1,12.7)$ \\
\hline 8 & 50.2 & $2.09(1 \mathrm{H}, \mathrm{d}, 7.63)$ & 50.0 & $2.11(1 \mathrm{H}, \mathrm{d}, 6.0)$ & 50.0 & $2.11(1 \mathrm{H}, \mathrm{d}, 6.0)$ \\
\hline 9 & 20.1 & / & 20.2 & / & 20.2 & / \\
\hline 10 & 26.4 & / & 26.4 & / & 26.4 & / \\
\hline 11 & 26.5 & $1.33(1 \mathrm{H}, \mathrm{m}, \mathrm{Ha}), 1.90(1 \mathrm{H}, \mathrm{m}, \mathrm{H} \beta)$ & 30.3 & $1.42(1 \mathrm{H}, \mathrm{m}, \mathrm{Ha}), 1.62(1 \mathrm{H}, \mathrm{m}, \mathrm{H} \beta)$ & 30.3 & $1.42(1 \mathrm{H}, \mathrm{m}, \mathrm{Ha}), 1.62(1 \mathrm{H}, \mathrm{m}, \mathrm{H} \beta)$ \\
\hline 12 & 32.3 & $1.60(1 \mathrm{H}, \mathrm{m}, \mathrm{Ha}), 1.68(1 \mathrm{H}, \mathrm{m}, \mathrm{H} \beta)$ & 32.4 & $1.60(1 \mathrm{H}, \mathrm{m}, \mathrm{Ha}), 1.69(1 \mathrm{H}, \mathrm{m}, \mathrm{H} \beta)$ & 32.4 & $1.60(1 \mathrm{H}, \mathrm{m}, \mathrm{Ha}), 1.69(1 \mathrm{H}, \mathrm{m}, \mathrm{H} \beta)$ \\
\hline 13 & 46.4 & / & 46.4 & / & 46.4 & / \\
\hline 14 & 45.3 & / & 45.3 & / & 45.3 & / \\
\hline 15 & 46.6 & $\begin{array}{l}1.64(1 \mathrm{H}, \mathrm{dd}, 5.0,7.5, \mathrm{H} \beta), 2.33(1 \mathrm{H} \\
\mathrm{dd}, 5.07 .5, \mathrm{Ha})\end{array}$ & 46.4 & $\begin{array}{l}1.63(1 \mathrm{H}, \mathrm{dd}, 5.2,7.6, \mathrm{H} \beta), 2.31(1 \mathrm{H}, \mathrm{dd} \\
5.2,7.6, \mathrm{Ha})\end{array}$ & 46.4 & $\begin{array}{l}1.63(1 \mathrm{H}, \mathrm{dd}, 5.2,7.6, \mathrm{H} \beta), 2.31(1 \mathrm{H}, \mathrm{dd}, \\
5.2,7.6, \mathrm{Ha})\end{array}$ \\
\hline 16 & 71.9 & $4.46(1 \mathrm{H}, \mathrm{ddd}, 5.0,7.6,7.8)$ & 72.3 & $4.43(1 \mathrm{H}, \mathrm{ddd}, 5.2,7.6,7.9)$ & 72.3 & $4.43(1 \mathrm{H}, \mathrm{ddd}, 5.2,7.6,7.9)$ \\
\hline 17 & 56.0 & $1.62(1 \mathrm{H}, \mathrm{dd}, 7.7,12.2)$ & 56.0 & $1.64(1 \mathrm{H}, \mathrm{dd}, 7.9,12.4)$ & 56.0 & $1.64(1 \mathrm{H}, \mathrm{dd}, 7.9,12.4)$ \\
\hline 18 & 16.2 & $1.18(3 \mathrm{H}, \mathrm{s})$ & 16.1 & $1.20(3 \mathrm{H}, \mathrm{s})$ & 16.1 & $1.20(3 \mathrm{H}, \mathrm{s})$ \\
\hline 19 & 24.9 & $\begin{array}{l}0.20(1 \mathrm{H}, \mathrm{d}, 4.5, \mathrm{Ha}) 0.85(1 \mathrm{H}, \mathrm{d}, 4.5 \\
\mathrm{H} \beta)\end{array}$ & 24.7 & $\begin{array}{l}0.18(1 \mathrm{H}, \mathrm{d}, 4.6, \mathrm{Ha}), 0.87(1 \mathrm{H}, \mathrm{d}, 4.6, \\
H \beta)\end{array}$ & 24.7 & $\begin{array}{l}0.18(1 \mathrm{H}, \mathrm{d}, 4.6, \mathrm{Ha}), 0.87(1 \mathrm{H}, \mathrm{d}, 4.6, \\
\mathrm{H} \beta)\end{array}$ \\
\hline 20 & 29.7 & $1.72(1 \mathrm{H}, \mathrm{m})$ & 28.5 & $1.95(1 \mathrm{H}, \mathrm{m})$ & 28.5 & $1.91(1 \mathrm{H}, \mathrm{m})$ \\
\hline 21 & 17.0 & $0.97(1 \mathrm{H}, \mathrm{d}, 7.6)$ & 17.2 & $0.97(1 \mathrm{H}, \mathrm{d}, 7.6)$ & 17.2 & $0.97(1 \mathrm{H}, \mathrm{d}, 7.6)$ \\
\hline 22 & 32.9 & $2.78(1 \mathrm{H}, \mathrm{m}, \mathrm{Ha}), 2.86(1 \mathrm{H}, \mathrm{m}, \mathrm{H} \beta)$ & 32.4 & $1.22(1 \mathrm{H}, \mathrm{m}, \mathrm{Ha}), 1.81(1 \mathrm{H}, \mathrm{m}, \mathrm{H} \beta)$ & 32.4 & $1.82(1 \mathrm{H}, \mathrm{m}, \mathrm{Ha}), 1.01(1 \mathrm{H}, \mathrm{m}, \mathrm{H} \beta)$ \\
\hline 23 & 29.5 & $1.21(1 \mathrm{H}, \mathrm{m}, \mathrm{Ha}), 1.97(1 \mathrm{H}, \mathrm{m}, \mathrm{H} \beta)$ & 30.3 & $1.42(1 \mathrm{H}, \mathrm{m}, \mathrm{Ha}), 1.62(1 \mathrm{H}, \mathrm{m}, \mathrm{H} \beta)$ & 31.3 & $2.12(1 \mathrm{H}, \mathrm{m}, \mathrm{Ha}), 1.12(1 \mathrm{H}, \mathrm{m}, \mathrm{H} \beta)$ \\
\hline 24 & 218.1 & / & 77.0 & $3.36(1 \mathrm{H}, \mathrm{dd}, 5.1,12.0)$ & 79.5 & $3.46(1 \mathrm{H}, \mathrm{dd}, 5.1,12.0)$ \\
\hline 25 & 76.5 & / & 71.9 & / & 71.9 & / \\
\hline 26 & 25.4 & $1.32(3 \mathrm{H}, \mathrm{s})$ & 25.4 & $1.32(3 \mathrm{H}, \mathrm{s})$ & 25.4 & $1.32(3 \mathrm{H}, \mathrm{s})$ \\
\hline 27 & 24.8 & $1.00(3 \mathrm{H}, \mathrm{s})$ & 24.0 & $1.18(3 \mathrm{H}, \mathrm{s})$ & 24.5 & $1.18(3 \mathrm{H}, \mathrm{s})$ \\
\hline 28 & 24.9 & $1.00(3 \mathrm{H}, \mathrm{s})$ & 24.9 & $1.00(3 \mathrm{H}, \mathrm{s})$ & 24.9 & $1.00(3 \mathrm{H}, \mathrm{s})$ \\
\hline 29 & 12.2 & $0.82(3 \mathrm{H}, \mathrm{s})$ & 12.8 & $0.82(3 \mathrm{H}, \mathrm{s})$ & 12.8 & $0.82(3 \mathrm{H}, \mathrm{s})$ \\
\hline 30 & 18.5 & $0.96(3 \mathrm{H}, \mathrm{s})$ & 18.5 & $0.94(3 \mathrm{H}, \mathrm{s})$ & 18.5 & $0.94(3 \mathrm{H}, \mathrm{s})$ \\
\hline $1^{\prime}$ & 102.6 & $4.36(1 \mathrm{H}, \mathrm{d}, 7.8)$ & 102.6 & $4.35(1 \mathrm{H}, \mathrm{d}, 7.7)$ & 102.5 & $4.35(1 \mathrm{H}, \mathrm{d}, 7.7)$ \\
\hline $2^{\prime}$ & 73.9 & $3.18(1 \mathrm{H}, \mathrm{dd}, 7.8,8.8)$ & 73.9 & $3.18(1 \mathrm{H}, \mathrm{dd}, 7.7,8.7)$ & 73.9 & $3.18(1 \mathrm{H}, \mathrm{dd}, 7.7,8.7)$ \\
\hline $3^{\prime}$ & 76.9 & $3.37(1 \mathrm{H}, \mathrm{dd}, 8.5,8.8)$ & 76.9 & $3.40(1 \mathrm{H}, \mathrm{dd}, 8.7,9.0)$ & 76.9 & $3.40(1 \mathrm{H}, \mathrm{dd}, 8.7,9.0)$ \\
\hline $4^{\prime}$ & 70.2 & $3.28(1 \mathrm{H}, \mathrm{dd}, 8.5,9.0)$ & 70.2 & $3.27(1 \mathrm{H}, \mathrm{dd}, 9.0,9.2)$ & 70.2 & $3.27(1 \mathrm{H}, \mathrm{dd}, 9.0,9.2)$ \\
\hline $5^{\prime}$ & 73.7 & $3.48(1 \mathrm{H}, \mathrm{m})$ & 73.7 & $3.48(1 \mathrm{H}, \mathrm{m})$ & 73.7 & $3.48(1 \mathrm{H}, \mathrm{m})$ \\
\hline $6^{\prime}$ & 63.9 & $\begin{array}{l}4.11(1 \mathrm{H}, \mathrm{dd}, 2.1,11.9), 4.48(1 \mathrm{H}, \mathrm{dd} \text {, } \\
2.1,11.9)\end{array}$ & 63.9 & $\begin{array}{l}4.11(1 \mathrm{H}, \mathrm{dd}, 2.0,12.0), 4.48(1 \mathrm{H}, \mathrm{dd}, \\
2.0,12)\end{array}$ & 63.9 & $\begin{array}{l}4.11(1 \mathrm{H}, \mathrm{dd}, 2.0,12.0), 4.48(1 \mathrm{H}, \mathrm{dd}, \\
2.0,12)\end{array}$ \\
\hline
\end{tabular}

\section{Statistical analysis}

Results are expressed as Mean \pm SEM (Standard Error of the Mean). One-way ANOVA followed by the Turkey's post test was used to compare the averages for the various groups in the antinociceptive tests in mice and in biochemical analysis. Two-way ANOVA followed by Bonferroni post-test was used to compare different groups in the antihyperalgesic and the inflammatory tests. These analyses were performed with the aid of Graph Pad Prism software version 5.0. 

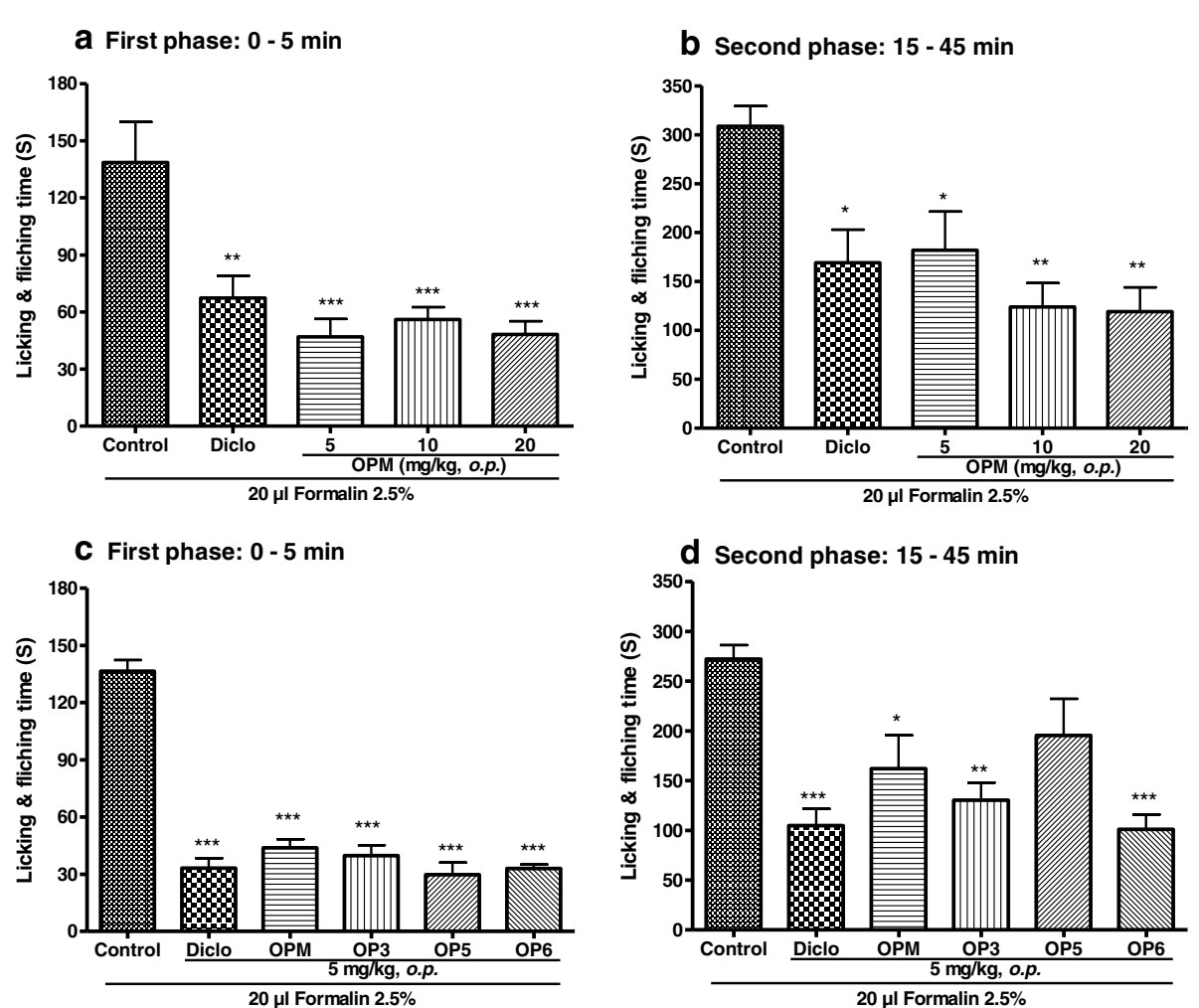

Fig. 3 Effect of mixed (OPM) and isolated (pallidioside $A: O P_{3}$, pallidioside $B: O P_{5}$ and pallidioside $C: O_{6}$ ) cycloartanes on the first (panels a and $\mathbf{c}$ ) and second (panels $\mathbf{b}$ and $\mathbf{d}$ ) phases of formalin-induced pain in mice. Each bar represents the mean $\pm \mathrm{SEM}, n=6$. ${ }^{*} P<0.05$, ${ }^{* *} p<0.01,{ }^{* * *} p<$ 0.001 significant difference as compared to control group. Diclo: diclofenac

\section{Results}

\section{Cycloartanes}

The individual main compounds are all characterized by the same basic structure and differ only on carbon 24 . This carbon atom, bearing one $\alpha-\mathrm{OH}$ in $\mathrm{OP}_{5}$ and one $\beta$ $\mathrm{OH}$ in $\mathrm{OP}_{6}$ respectively, was oxidized in ketone in molecule $\mathrm{OP}_{3}$ (Fig. 1). ${ }^{1} \mathrm{H}$ and ${ }^{13} \mathrm{C}$ data of these compounds were previously described by Tigoufack et al. [15].

The aglycons derivatives generally have the same basic structure as the main compound from which they derived except the fact that, carbon atom $\mathrm{C}-7$ which was linked to a glucosyl moiety in each precursor, was now linked to a $\beta-\mathrm{OH}$ group as indicated in Fig. 2.

$\mathrm{AOP}_{1}$ was obtained as a white powder. $[\alpha] \mathrm{D}_{23}=+85$ $\left(\mathrm{CH}_{3} \mathrm{OH}, c=0.92\right)$; IR $(\mathrm{NaCl}) v_{\max }\left(\mathrm{cm}^{-1}\right): 3375(\mathrm{OH})$, $1703(\mathrm{C}=\mathrm{O}), 1246(\mathrm{C}-\mathrm{O}),{ }^{1} \mathrm{H}$ and ${ }^{13} \mathrm{C}$ NMR data see Table 1. HR-TOFESIMS $m / z: 513.3717[\mathrm{M}+\mathrm{Na}]^{+}$(calcd. For $\mathrm{C}_{30} \mathrm{H}_{50} \mathrm{O}_{5} \mathrm{Na}, 513.3712$ ).

$\mathrm{AOP}_{2}$ was obtained as a white powder. $[\alpha] \mathrm{D}_{23}=+58.6$ $\left(\mathrm{CH}_{3} \mathrm{OH}, c=0.89\right)$; IR $(\mathrm{NaCl}) v_{\max }\left(\mathrm{cm}^{-1}\right): 3465(\mathrm{OH})$, $1249(\mathrm{C}-\mathrm{O}),{ }^{1} \mathrm{H}$ and ${ }^{13} \mathrm{C}$ data, see Table 1 ; HRTOFESIMS $m / z: 515.3719 \quad[\mathrm{M}+\mathrm{Na}]^{+}$(calcd. For $\mathrm{C}_{30} \mathrm{H}_{52} \mathrm{O}_{5} \mathrm{Na}, 515.3712$ ).

$\mathrm{AOP}_{3}$ was obtained as a white powder. $[\alpha] \mathrm{D}_{23}=+34.2$ $\left(\mathrm{CH}_{3} \mathrm{OH}, c=1.20\right)$; IR $(\mathrm{NaCl}) v_{\max }\left(\mathrm{cm}^{-1}\right): 3477(\mathrm{OH})$,
1250 (C-O), ${ }^{1} \mathrm{H}$ and ${ }^{13} \mathrm{C}$ data, see Table 1; HRTOFESIMS $m / z: 515.3719 \quad[\mathrm{M}+\mathrm{Na}]^{+}$(calcd. For $\mathrm{C}_{30} \mathrm{H}_{52} \mathrm{O}_{5} \mathrm{Na}$, 515.3712).

The acetylated derivatives generally have the same basic structure as the main compound from which they derived except the fact that all primary and secondary hydroxyl groups were acetylated as showed in Fig. 2.

$\mathrm{HOP}_{1}$ was obtained as a white powder. $[\alpha] \mathrm{D}_{23}=+18.2$ $\left(\mathrm{CH}_{3} \mathrm{OH}, c=0.482 \times 10^{-2}\right)$; IR $(\mathrm{NaCl}) v_{\max }\left(\mathrm{cm}^{-1}\right): 3450$ $(\mathrm{OH}), 1249(\mathrm{C}-\mathrm{O}), 1715(\mathrm{C}=\mathrm{O}) .{ }^{1} \mathrm{H}$ and ${ }^{13} \mathrm{C}$ data, see Table 2.

$\mathrm{HOP}_{2}$ was obtained as a white powder. $[\alpha] \mathrm{D}_{23}=+34.2$ $\left(\mathrm{CH}_{3} \mathrm{OH}, c=0.487 \times 10^{-2}\right) ; \mathrm{IR}(\mathrm{NaCl}) v_{\max }\left(\mathrm{cm}^{-1}\right): 3450$ $(\mathrm{OH}), 1250(\mathrm{C}-\mathrm{O}), 1713(\mathrm{C}=\mathrm{O}) .{ }^{1} \mathrm{H}$ and ${ }^{13} \mathrm{C}$ data, see Table 2.

$\mathrm{HOP}_{3}$ was obtained as a white powder. $[\alpha] \mathrm{D}_{23}=+20.2$ $\left(\mathrm{CH}_{3} \mathrm{OH}, c=0.458 \times 10^{-2}\right)$; IR $(\mathrm{NaCl}) v_{\max }\left(\mathrm{cm}^{-1}\right): 3477$ $(\mathrm{OH}), 1249(\mathrm{C}-\mathrm{O}), 1718(\mathrm{C}=\mathrm{O}) .{ }^{1} \mathrm{H}$ and ${ }^{13} \mathrm{C}$ data, see Table 2.

\section{Effect of the mixture and isolated cycloartanes on formalin-induced pain in mice}

Treatment with different doses of the mixture of cycloartanes significantly reduced the pain behavior 


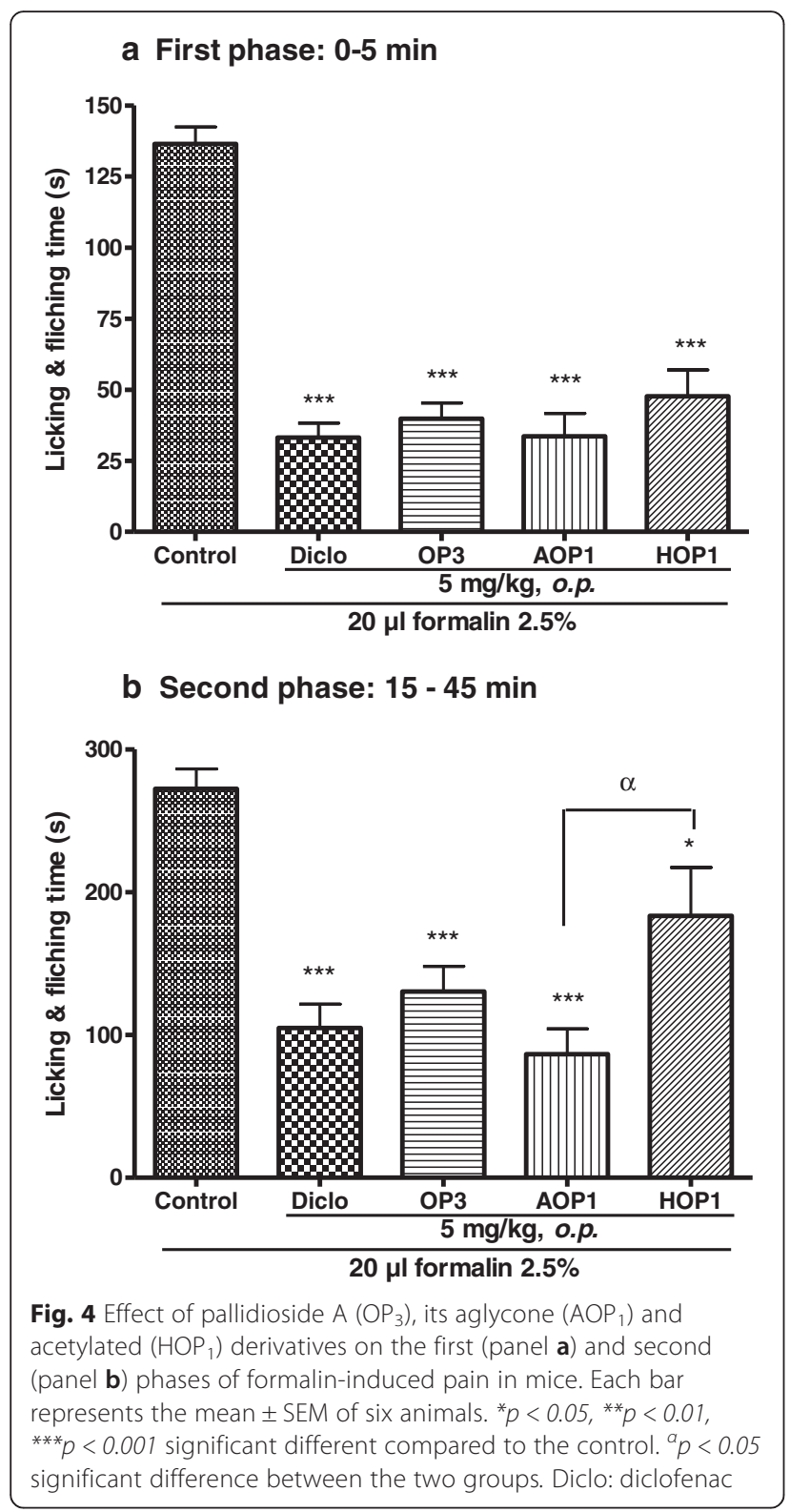

during the first and the second phases of pain induced by formalin as compared to the control group. The percentages of inhibition ranged from 51.33 to $65.15 \%$ for the first phase (Fig. 3a) and from 45.15 to $61.42 \%$ for the second phase (Fig. 3b). The highest percentages of inhibition were obtained at $5 \mathrm{mg} / \mathrm{kg}$ during the first phase and $20 \mathrm{mg} / \mathrm{kg}$ in the second. Concordantly, systemic oral administration of the three main compounds $\left(\mathrm{OP}_{3}, \mathrm{OP}_{5}\right.$ and $\left.\mathrm{OP}_{6}\right)$ significantly $(p<0.001)$ reduced the licking time of the paw during the first phase. No significant difference was observed between the effect of mixture and the individual compounds (Fig. 3c). $\mathrm{OP}_{5}$ reduced pain behavior by about $13 \%$ during the second phase but this effect missed a First phase: 0 - $5 \mathrm{~min}$

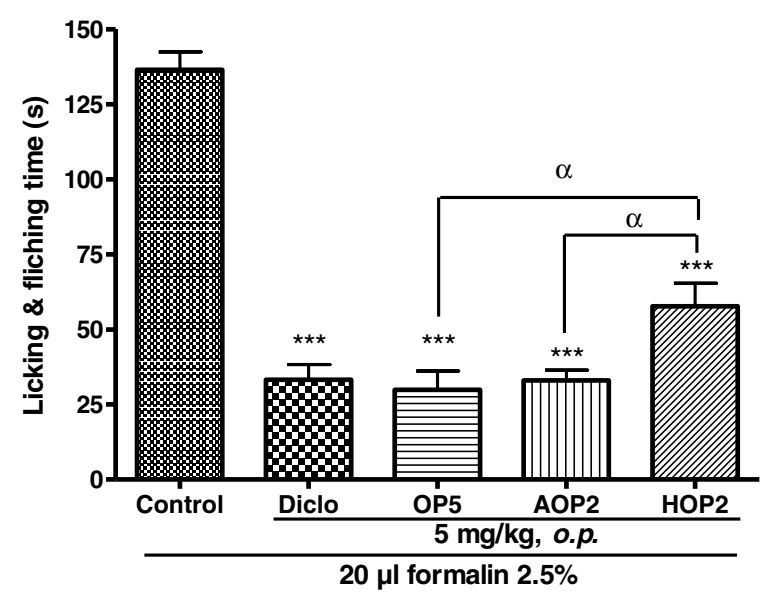

b Second phase: 15 - $45 \mathrm{~min}$

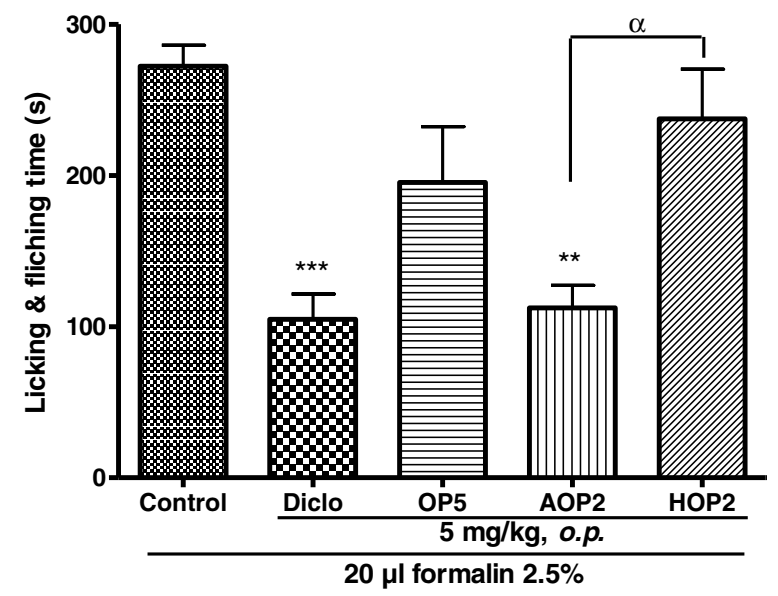

Fig. 5 Effect of pallidioside $B\left(O P_{5}\right)$, its aglycone $\left(A O P_{2}\right)$ and acetylated $\left(\mathrm{HOP}_{2}\right)$ derivatives on the first (panel $\mathbf{a}$ ) and second (panel $\mathbf{b}$ ) phases of formalin-induced pain in mice. Each bar represents the mean $\pm \mathrm{SEM}$, $n=6$. ${ }^{* *} P<0.01,{ }^{* *} p<0.001$ significant difference compared to control. ${ }^{a} p<0.05$ significant difference between the two groups. Diclo: diclofenac

significance $(p>0.05)$, while $\mathrm{OP}_{3}$ and $\mathrm{OP}_{6}$ exhibited significant analgesic effect similar to that of diclofenac (Fig. 3d).

Effect of $\mathrm{OP}_{3}$, its aglycone $\left(\mathrm{AOP}_{1}\right)$ and acetylated $\left(\mathrm{HOP}_{1}\right)$ derivatives on formalin-induced pain in mice

As shown in Fig. 4, oral administration of $\mathrm{OP}_{3}$ and its derivatives exhibited an effective analgesic effect on both phases of pain. There was no significant difference between the effect of $\mathrm{OP}_{3}$ and its derivatives during the first phase (Fig. 4a). At the second phase $\mathrm{OP}_{3}$ and derivatives also showed a significant analgesic effect but a significant difference was observed between the effect of the two $\mathrm{OP}_{3}$ derivatives, the aglycone $\mathrm{AOP}_{1}$ being 


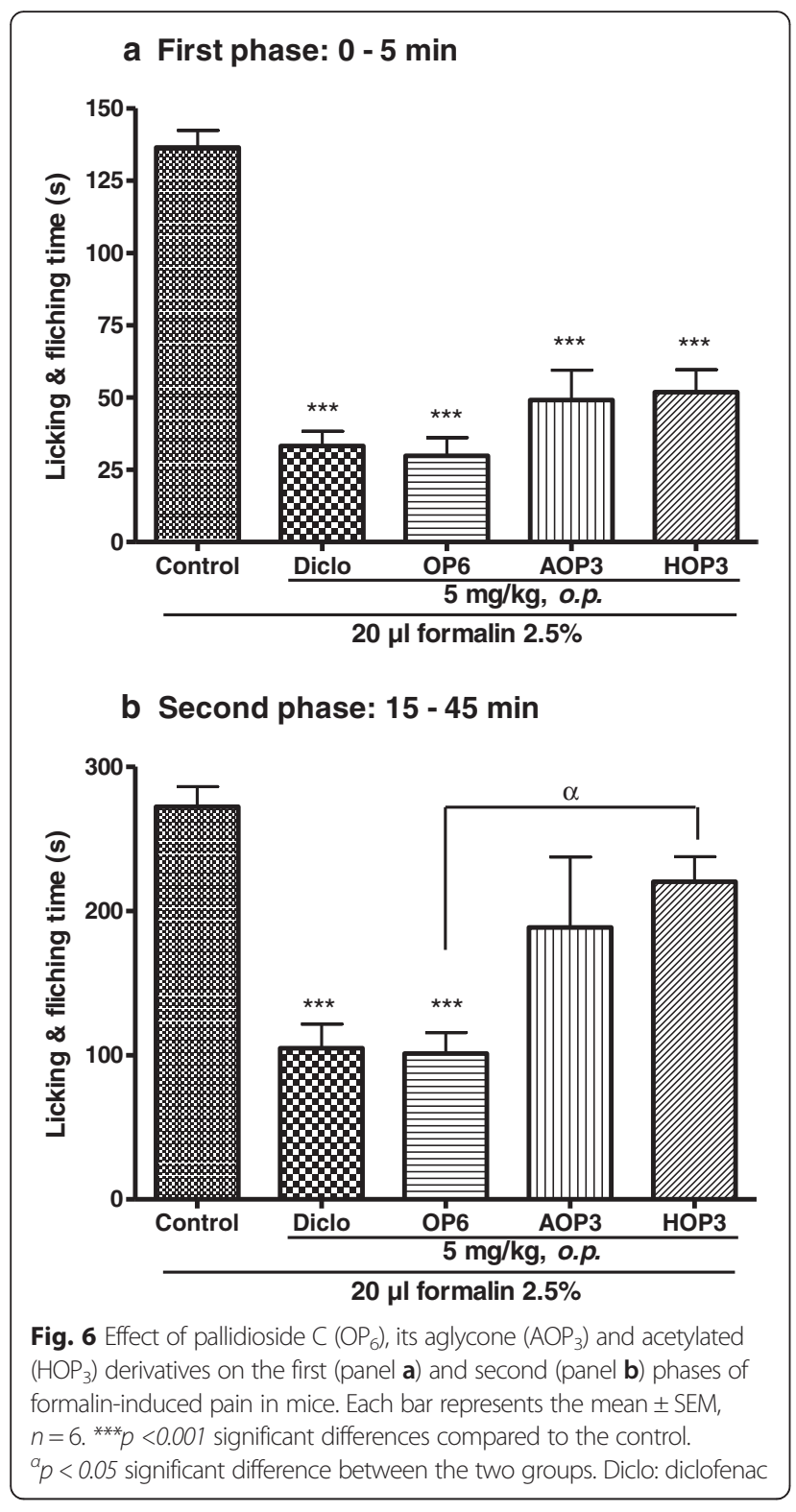

significantly $(p<0.05)$ more active than the acetylated $\mathrm{HOP}_{1}$ (Fig. 4b).

Effect of $\mathrm{OP}_{5}$, its aglycone $\left(\mathrm{AOP}_{2}\right)$ and acetylated $\left(\mathrm{HOP}_{2}\right)$ derivatives on formalin-induced pain in mice

Although $\mathrm{OP}_{5}$ and its derivatives all induced a significant analgesic effect on the first phase of pain induced by intraplantar administration of formalin, the acetylated derivative was significantly less potent than $\mathrm{OP}_{5}$ and its aglycone (Fig. 5a). On the second phase, $\mathrm{OP}_{5}$ and its acetylated derivative did not show significant analgesic effect as compared to control but $\mathrm{AOP}_{2}$ was significantly active as compared both to the control and $\mathrm{HOP}_{2}$ treated groups (Fig. 5b).
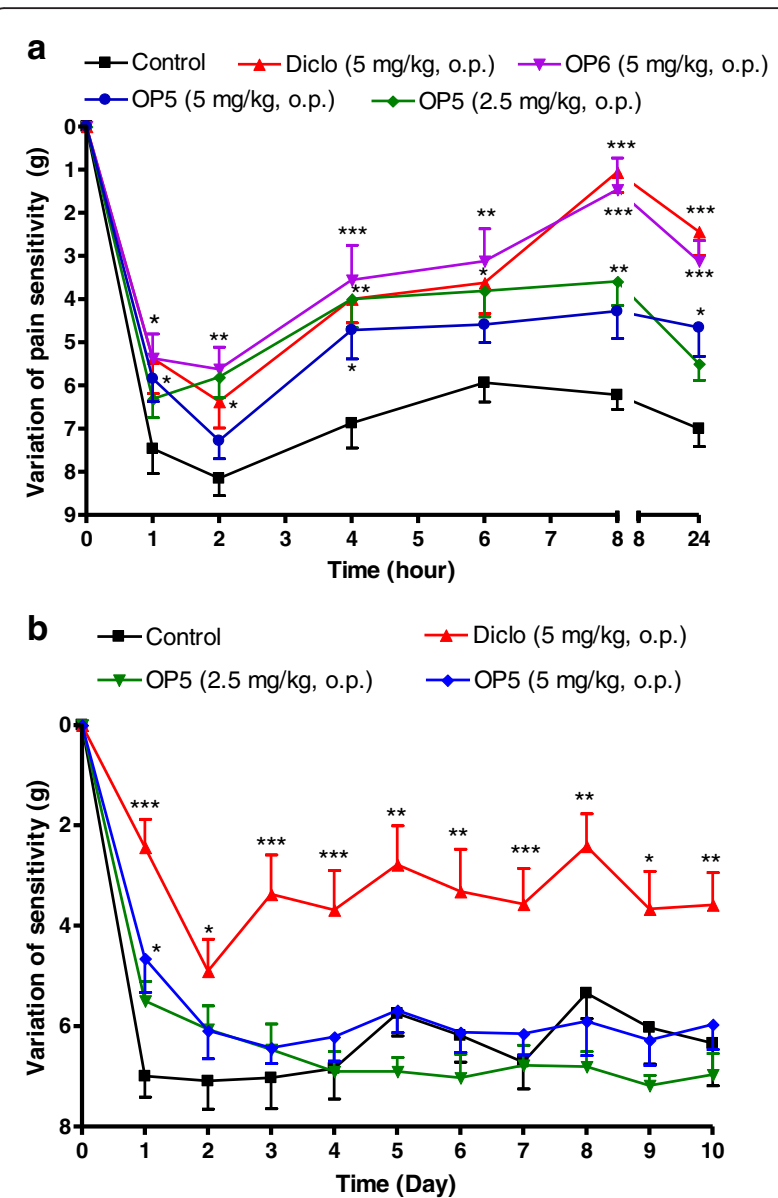

Fig. 7 Time-dependant acute (panel a) effect of Pallidioside $B\left(O_{5}\right)$ and Pallidioside $\mathrm{C}\left(\mathrm{OP}_{6}\right)$ and repeated treatment of $\mathrm{OP}_{5}$ (panel b) on mechanical pain induced by Randal sellito in rat paw injected with formalin $(2 \%, 100 \mu \mathrm{l})$. Animals were orally treated with different compounds and injected one hour later in the paw with formalin. Pain threshold was measured before treatment and after formalin injection. For chronic experiment, animals were daily treated and pain threshold was measured before drug administration. Each point represents the mean \pm SEM,$n=8$. ${ }^{*} p<0.05,{ }^{* *} p<0.01,{ }^{* * *} p<0.001$ significant differences compared to the control. Diclo: diclofenac

Effect of $\mathrm{OP}_{6}$, its aglycone $\left(\mathrm{AOP}_{3}\right)$ and acetylated $\left(\mathrm{HOP}_{3}\right)$ derivatives on formalin-induced pain in mice

$\mathrm{OP}_{6}$ and its two derivatives exhibited significant analgesic effect on the first phase of pain induced by formalin. Meanwhile, $\mathrm{OP}_{6}$ proved to be more effective than any of its derivatives (Fig. 6a). This was more marked at the second phase were $\mathrm{AOP}_{3}$ and $\mathrm{HOP}_{3}$ lacked significant activity. However, $\mathrm{OP}_{6}$ was significantly active as compared both to the control group and the $\mathrm{HOP}_{3}$ treated group (Fig. 6b).

Effect of $\mathrm{OP}_{5}$ and $\mathrm{OP}_{6}$ on mechanical hyperalgesia and inflammation induced by formalin in rats

Systemic oral administration of $\mathrm{OP}_{5}$ and $\mathrm{OP}_{6}$ one hour before induction of pain both produced significant 


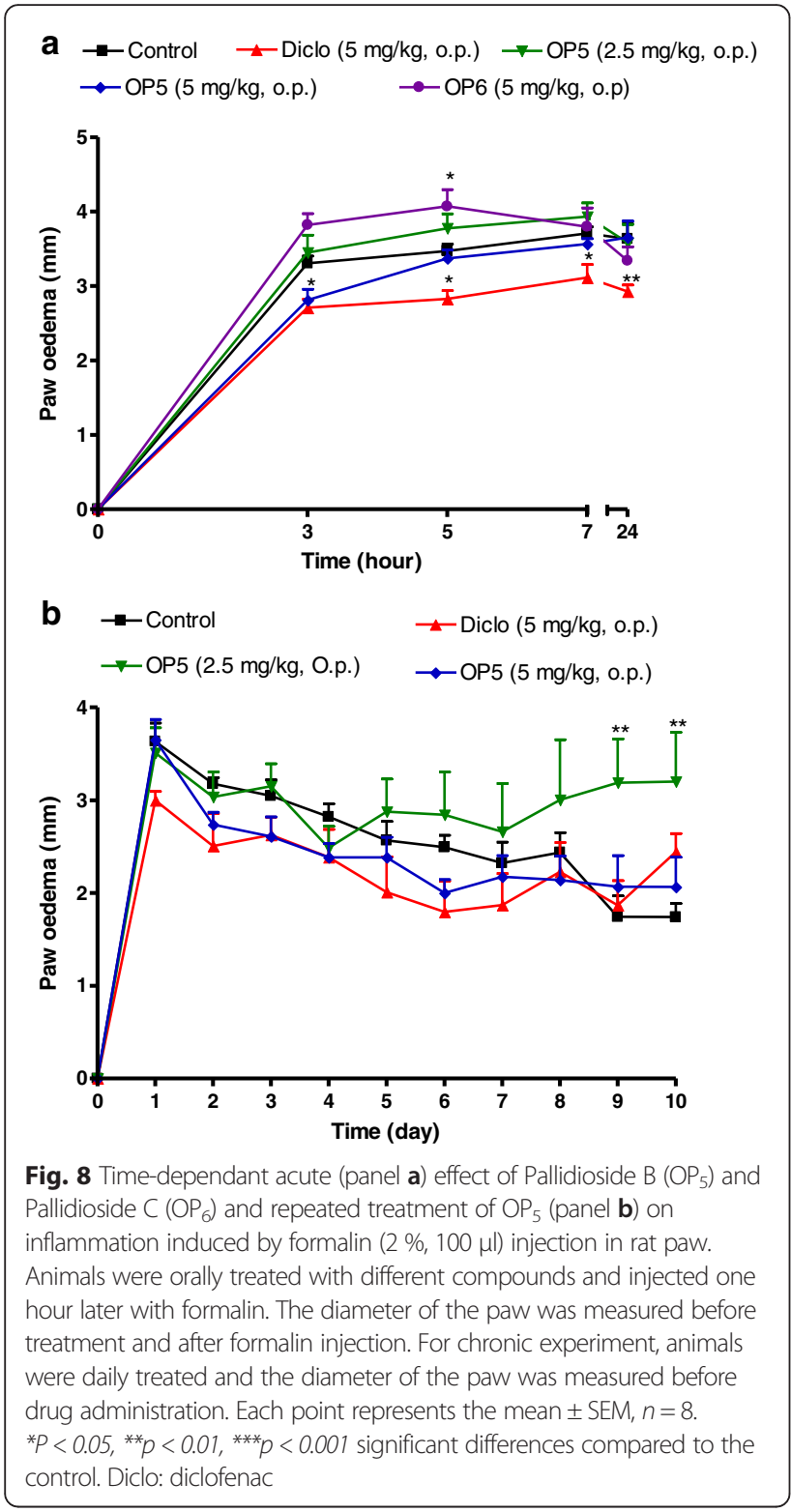

inhibition of hyperalgesia for up to $24 \mathrm{~h}$. The acute maximal effect was observed $8 \mathrm{~h}$ post induction with $\mathrm{OP}_{6}$ at the dose of $5 \mathrm{mg} / \mathrm{kg}$ as compared to the negative control (Fig. 7a). This effect was similar to that of diclofenac at the same dose. During the 10 days treatment, $\mathrm{OP}_{5}$ at both doses shows significant effect only at the first day (Fig. 7b).

As shown in Fig. 8, $\mathrm{OP}_{6}$ instead increase the inflammation induced by formalin. The inflammation was significantly reduced by $\mathrm{OP}_{5}$ at the dose of $5 \mathrm{mg} / \mathrm{kg}$ at the third hour post induction. Repeated administration of $\mathrm{OP}_{5}$ at both doses did not show any anti-inflammatory activity all along the 10 days of experiment.

\section{Biochemical analyses}

Results of the assays of oxidative stress parameters are presented in Table 3. After 10 days of repeated treatment, $\mathrm{OP}_{5}$ significantly reduced the malondialdehyde production and increased the superoxide dismutase activity in the liver, but has no effect on these parameters in the kidney. $\mathrm{OP}_{5}$ did not affect the concentration of $\mathrm{NO}$, glutathione and the activity of catalase both in the liver and kidneys. The concentration of ALAT dosedependently increased in the serum of $\mathrm{OP}_{5}$ treated animals as compared to the control group. Meanwhile, the effect was not statistically significant.

\section{Ulcerogenic activity}

It is observed from Table 4 that after 10 days treatment, animals treated with $\mathrm{OP}_{5}$ at both doses showed no ulceration in their gastric mucosa. Ulceration was recorded in all animal treated with diclofenac with a percentage ulcerated surface of $15.95 \%$. It was also observed that repeated administration of diclofenac at the dose of $5 \mathrm{mg} / \mathrm{kg} /$ day significantly reduced the mucus production, whereas $\mathrm{OP}_{5}$ has no effect.

\section{Discussion}

This work was undertaken to evaluate the analgesic and anti-inflammatory effects of three cycloartanes isolated from Oxyanthus pallidus and their derivatives. The mixture of cycloartanes significantly inhibited both phases of pain induced by the injection of formalin. When tested individually, isolated compounds exhibited different intensities of activity. During the first phase of pain, the three compounds showed significant antinociceptive effect. No significant difference was observed between their effects although $\mathrm{OP}_{5}$ was nominally the most effective. Regarding the second phase of pain, $\mathrm{OP}_{3}$ and $\mathrm{OP}_{6}$ induced a significant analgesic effect while $\mathrm{OP}_{5}$ showed less activity. It is well known that formalin model which is consider mimicking clinical pain [23], has two main distinct phases. The first one characterized as neurogenic pain is related to chemical stimulation of $\mathrm{A}_{\delta}$ and $\mathrm{C}$ fibers, the release of excitatory amino-acids, nitric oxide and substance $\mathrm{P}$ while the second phase known as inflammatory pain is related to the release of bradykinin and prostaglandins $[16,24]$. Drugs that inhibit only the first or both phases are considered as central analgesics while those acting only at the second phase are considered as peripheral analgesic drugs [25]. The mixture of cycloartanes as well as $\mathrm{OP}_{3}$ and $\mathrm{OP}_{6}$ significantly inhibited both phases of formalin-induced pain while $\mathrm{OP}_{5}$ inhibited predominately the first phase. The activity of these compounds either taken individually or as a mixture was significantly more potent at the first phase than at the second. These results indicate that the tested cycloartanes may be central acting molecules. The 
Table 3 Effects of different treatments on the biochemical parameters of oxidative stress and hepatotoxicity

\begin{tabular}{llcccc}
\hline Parameters & Tissue & Control & Diclofenac $(5 \mathrm{mg} / \mathrm{kg})$ & OP5 $(2.5 \mathrm{mg} / \mathrm{kg})$ & OP5 $(5 \mathrm{mg} / \mathrm{kg})$ \\
\hline NO $(\mu \mathrm{mol} / \mathrm{g}$ tissue) & liver & $0.49 \pm 0.06$ & $0.28 \pm 0.06^{*}$ & $0.48 \pm 0.04$ & $0.36 \pm 0.03$ \\
& kidney & $1.014 \pm 0.11$ & $0.66 \pm 0.15$ & $1.23 \pm 0.15$ & $0.85 \pm 0.06$ \\
Glutathione $(\mu \mathrm{mol} / \mathrm{g}$ tissue) & liver & $0.12 \pm 0.01$ & $0.11 \pm 0.01$ & $0.12 \pm 0.01$ & $0.10 \pm 0.01$ \\
& kidney & $0.07 \pm 0.01$ & $0.04 \pm 0.01$ & $0.09 \pm 0.01$ & $0.07 \pm 0.00$ \\
MDA ( $\mu \mathrm{mol} / \mathrm{g}$ tissue) & liver & $9.37 \pm 0.74$ & $9.80 \pm 0.46$ & $3.10 \pm 0.37^{* * *}$ & $9.42 \pm 0.24$ \\
& kidney & $0.12 \pm 0.01$ & $0.09 \pm 0.02$ & $0.14 \pm 0.01$ & $0.12 \pm 0.00$ \\
SOD (U/mg protein) & liver & $0.05 \pm 0.01$ & $0.12 \pm 0.00^{*}$ & $0.08 \pm 0.02$ & $0.11 \pm 0.01^{*}$ \\
& kidney & $0.19 \pm 0.08$ & $0.12 \pm 0.05$ & $0.09 \pm 0.02$ & $0.10 \pm 0.04$ \\
Catalase (U/mg protein) & kidney & $0.08 \pm 0.01$ & $0.08 \pm 0.01$ & $0.10 \pm 0.02$ & $0.08 \pm 0.01$ \\
ALAT (U/mg protein) & serum & $0.39 \pm 0.15$ & $0.4 \pm 0.01$ & $0.60 \pm 0.08$ & $0.80 \pm 0.21$ \\
ASAT (U/mg protein) & serum & $1.28 \pm 0.10$ & $1.58 \pm 0.13$ & $1.35 \pm 0.07$ & $1.313 \pm 0.11$ \\
\hline
\end{tabular}

Each value represents the average \pm ESM of eight animals. $p<0.05,{ }^{* * *} p<0.001$ significant difference compared to the control group

structural difference between these compounds is at C24 , which in the case of $\mathrm{OP}_{3}$ carries a ketone while that of $\mathrm{OP}_{5}$ and $\mathrm{OP}_{6}$ carry a hydroxyl group. The combination of the structure and activity of molecules shows that the substitution on $\mathrm{C}-24$ by the ketone group or the hydroxyl group does not significantly affect the analgesic activity in the formalin model. However, the analgesic activity on the inflammatory pain can be improved if the hydroxyl group at C-24 is placed in front of the plane.

Acid hydrolysis leading to aglycone did not affect the analgesic activity of different compounds on the neurogenic pain but significantly reduced the effect of $\mathrm{OP}_{6}$ on the inflammatory pain. These results suggest a probable interaction between the hydroxyl group and sugar, all placed in front of the plane, which could be necessary for the activity of $\mathrm{OP}_{6}$. This hypothesis is further supported by the results obtained after acetylating $\mathrm{OP}_{3}, \mathrm{OP}_{5}$ and $\mathrm{OP}_{6}$. In fact, the process has significantly inhibited the analgesic activity of all these compounds. We can then conclude that the hydroxyl groups attached to the aglycone are responsible for the analgesic activity of these cycloartanes, mainly as far as the inflammatory pain is concern. These results corroborate those previously obtained by Ayatollahi et al. [12] who showed that cycloartanes can deactivate protein Kinase C, thanks to their hydroxyl group. In addition, it has been shown that PKC mediates the phase 2 of formalin-induced pain [26]. Taking all together, it can be thought that cycloartanes from Oxyanthus pallidus induced their analgesic effect by inhibiting at least partially the activation of PKC.

$\mathrm{OP}_{5}$ and $\mathrm{OP}_{6}$ were further tested on mechanical hyperalgesia and inflammation induced by formalin in rats. In the acute test, they significantly reduced mechanical hyperalgesia from the first hour after induction of pain and lasted until the 24th hour post administration. $\mathrm{OP}_{5}$, in contrary of $\mathrm{OP}_{6}$, showed a significant anti-inflammatory effect only at the 3rd hour post treatment. These results indicate that the analgesic effects of tested cycloartanes are not dependent to their anti-inflammatory activity if any. Furthermore, repeated treatment with $\mathrm{OP}_{5}$ did not show any anti-inflammatory effect, demonstrating that $\mathrm{OP}_{5}$ is devoid of anti-inflammatory effect.

It was observed that unlike diclofenac, repeated oral administration of $\mathrm{OP}_{5}$ did not induce gastric ulceration and did not significantly affect the gastric mucus weight. Diclofenac reduces mucus production and induces gastric ulcer through the reduction of prostaglandin resulting from its inhibitory effect on cyclooxygenase. The above result suggests that $\mathrm{OP}_{5}$ do not inhibit cyclooxygenase.

The strong involvement of oxidative stress in inflammatory and painful phenomena $[27,28]$ has motivated the study of the in vivo antioxidant activity of $\mathrm{OP}_{5}$ in animals treated chronically. $\mathrm{OP}_{5}$ significantly reduced malondialdehyde (MDA) and increased the level of superoxide dismutase (SOD) without affecting the activity of catalase and nitric oxide amount. These results suggest that $\mathrm{OP}_{5}$

Table 4 Evaluation of the ulcerogenic activity of Diclofenac (Diclo) and $\mathrm{OP}_{5}$

\begin{tabular}{llllll}
\hline Groups & Doses $(\mathrm{mg} / \mathrm{kg})$ & Mucus weight $(\mathrm{mg})$ & Total surface of stomach $\left(\mathrm{mm}^{2}\right)$ & ulcerated surface $\left(\mathrm{mm}^{2}\right)$ & $\%$ of ulcerated animals \\
\hline Control & & $0.163 \pm 0.013$ & $1479.00 \pm 72.79$ & $0.00 \pm 0.00$ & 0 \\
Diclo & 5 & $0.073 \pm 0.014^{*}$ & $1406.00 \pm 78.92$ & $221.10 \pm 0.00^{* * *}$ & 100 \\
OP5 & 2,5 & $0.127 \pm 0.018$ & $1886.00 \pm 127.00$ & $0.00 \pm 0.00$ & 0 \\
OP5 & 5 & $0.090 \pm 0.009$ & $1723.00 \pm 46.90$ & $0.00 \pm 0.00$ & 0
\end{tabular}

Each value represents the mean \pm SEM of eight animals. ${ }^{*} p<0.05,{ }^{* * *} p<0.001$ significant difference compared to the control group. Diclo diclofenac 
might potentiate the in vivo antioxidant capacity of the organism by increasing the SOD activity that could lead to reduction of lipid peroxydation. In other to evaluate the impact of $\mathrm{OP}_{5}$ on the liver integrity, alanine aminotrasferase (ALAT) and aspartate aminotrasferase (ASAT) activities were assayed in the serum. A dose dependent increase in ALAT was observed in $\mathrm{OP}_{5}$ treated group, although not significant. It is well known that plasma increase in ALAT and ASAT, especially ALAT is a result of liver cells necrosis. Thus, even though there was no evidence of hepatotoxic effect, caution should be taken when using $\mathrm{OP}_{5}$.

\section{Conclusion}

Cycloartanes isolated from Oxyanthus pallidus possess analgesic effects that are dissociated from anti-inflammatory activity if any. The analgesic effect of these compounds especially the effect on inflammatory pain may be due to the presence of hydroxyl group in front of the plane. Meanwhile, these cycloartanes are more potent on the neurogenic than on the inflammatory pain.

\section{Competing interests}

The authors declare that they have no competing interests.

\section{Authors' contribution}

TBN and DN designed the work. BNP, IN and ASA conducted the work and collected data. TBN and PW analysed the data. BNP drafted the manuscript and TBN, DN, WG and RDT revised it critically. All authors agree to be accountable for all aspects of the work. All authors read and approved the final manuscript.

\section{Acknowledgement}

This research work was partially supported by the Alexander von Humboldt Foundation (Germany) to which the authors express their profound gratitude.

\section{Author details}

'Laboratory of Animal Physiology and Phytopharmacology, Department of Animal Biology, Faculty of Science, University of Dschang, P.O. Box 67, Dschang, Cameroon. 'Laboratory of Applied and Environmental Chemistry, Department of Chemistry, Faculty of Science, University of Dschang, P.O. Box 67, Dschang, Cameroon. ${ }^{3}$ Department of Neurophysiology, Centre for Biomedicine and Medical Technology Mannheim (CBTM), Heidelberg University, Ludolf-Krehl-Str. 13, D-68167 Mannheim, Germany.

Received: 27 August 2015 Accepted: 3 March 2016

Published online: 09 March 2016

\section{References}

1. Clère F, Perriot M, Henry F, Kipper MC, Alcalay V, Voisine T. Enquête de prévalence de la douleur chez les patients hospitalisés au centre hospitalier de Châteauroux. Douleurs. 2007;8:67.

2. Chikanza IC, Kozaci DL. Corticosteroid resistance in rheumatoid arthritis: molecular and cellular perspectives. Rheumatology. 2004:43:1337-45.

3. Michetti P, Mottet C, Juillerat P, Felley C, Vader JP, Burnand B, et al. Severe and steroid-resistant Crohn's disease. Digestion. 2005;71:19-25.

4. Hollinshead SP, Astles PC, Chambers MG, Johnson MP, Palmer J, Tidwell MW. Discovery and optimization of novel purines as potent and selective CB2 agonists. Bioorg Med Chem Lett. 2012;22:4962-6.

5. Varamini P, Hussein WM, Mansfeld FM, Toth I. Synthesis, biological activity and structure-activity relationship of endomorphin-1/substance $P$ derivatives. Bioorg Med Chem Lett. 2012;20:6335-43.

6. Lee YS, Hall SM, Ramos-Colon C, Remesic M, LeBaron L, Nguyen A, Rankin D, Porreca F, Lai J, Hruby VJ. Modification of amphipathic non-opioid dynorphin A analogues for rat brain bradykinin receptors. Bioorg Med Chem Lett. 2015;25:30-3.
7. Chicca A, Marazzi J, Gertsch J. The antinociceptive triterpene $\beta$-amyrin inhibits 2-arachidonoylglycerol (2-AG) hydrolysis without directly targeting cannabinoid receptors. Br J Pharmacol. 2012;167:1596-608.

8. Longhi-Balbinot DT, Lanznaster D, Baggio $\mathrm{CH}$, Silva MD, Cabrera $\mathrm{CH}$, Facundo VA, et al. Anti-inflammatory effect of triterpene $3 \beta, 6 \beta, 16 \beta$ trihydroxylup-20(29)-ene obtained from Combretum leprosum Mart \& Eich in mice. J Ethnopharmacol. 2012;142:59-64.

9. Koneri RB, Samaddar S, Simi SM, Rao ST. Neuroprotective effect of a triterpenoid saponin isolated from Momordica cymbalaria Fenzl in diabetic peripheral neuropathy. Indian J Pharmacol. 2014;46:76-81.

10. Le Borgne M, Duflos M, Le Baut G, Nicholls PJ, Hartmann RW. Dérivés indoliques à activités anti-inflammatoire ou antitumorale. Ann Pharm Fr. 2000;58:316-20.

11. Tian Z, Xiao PG, Wen J, Huang F, Yang MS, Chen S. Review of bioactivities of natural cycloartane triterpenoids. ZhongguoZhong Yao ZaZhi. 2006;31:625-9.

12. Ayatollahi AM, Ghanadian M, Afsharypuor S, Mesaik MA, Abdalla OM, Shahlaei M, et al. Cycloartanes from Euphorbia aellenii Rech. f. and their Antiproliferative Activity. Iranian J Pharmaceut Res. 2011;10:105-12.

13. De Souza Nunes JP, da Silva KA, da Silva GF, Quintão NL, Corrêa R, CechinelFilho $V$, et al. The antihypersensitive and antiinflammatory activities of a benzofuranone derivative in different experimental models in mice: the importance of the protein kinase C pathway. Anesth Analg. 2014;1 19:836-46.

14. Wang S, Joseph J, Ro JY, Chung MK. Modality-specific mechanisms of PKC induced hypersensitivity of TRPV1: S800 is a polymodal sensitization site. Pain. 2015;156:931-41.

15. Tigoufack IB, Ngnokam D, Tapondjou LA, Harakat D, Voutquenne L. Cycloartane glycosides from leaves of Oxyanthus pallidus. Phytochem. 2010;71:2182-6.

16. Nguelefack TB, Nana P, Atsamo AD, Dimo T, Watcho P, Dongmo AB, et al. Analgesic and anticonvulsant effects of extracts from the leaves of Kalanchoe crenata (Andrews) Haworth (Crassulaceae). J Ethnopharmacol. 2006;106:70-5.

17. Mandegary A, Sayyah M, Mahmood RH. Antinociceptive and antiinflammatory activity of the seed and root extracts of ferula gummosaboiss in mice and rats. DARU. 2004;2:58-61.

18. Nguelefack TB, Feumebo CB, Ateufack G, Watcho P, Tatsimo S, Atsamo AD, Tane $P$, Kamanyi A. Anti-ulcerogenic properties of the aqueous and methanol extracts from the leaves of Solanum torvum Swartz (Solanaceae) in rats. J Ethnopharmacol. 2008;119:135-40.

19. Misra HP, Fridovich I. The role of superoxide anion in the autoxidation of epinephrine and a simple assay for superoxide dismutase. J Boil Chem. 1972;14:3170-5.

20. Ohkawa H, Ohishi N, Yagi K. Assay for lipid peroxides in animal tissues by thiobarbituric acid reaction. Anal Biochem. 1979;95(2):351-8.

21. Dimo T, Tsala DE, Dzeufiet PD, Penlap BV, Njifutie N. Effects of Aliafia multiflora Stapf on lipid peroxidation and antioxidant enzyme status in carbon tetrachloride-treated rats. Pharmacologyonline. 2006;2:76-86.

22. Fiddler RM. Collaborative study of modified method of analysis for nitrite in meat and meat product. J AOAC. 1977;60:594-9.

23. Tjölsen A, Hole K. Animals models of analgesia. In: Dickenson AH, Besson JM, editors. The Pharmacology of Pain; Handbook of Experimental Pharmacology Series, v. 25. Springer-Verlag: Berlin; 1997. p. 1.

24. Hunskaar S, Hole K. The formalin test in mice: Dissociation between inflammatory and non-inflammatory pain. Pain. 1987;30:103-4.

25. Queiroz RB, Carvalho FL, Fonseca DV, Barbosa-Filho JM, Salgado PRR, Paulo $L L$, et al. Antinociceptive effect of hydantoin 3-Phenyl-5-(4-ethylphenyl)imidazolidine-2,4-dione in Mice. Molecules. 2015;20:974-86.

26. Sweitzer SM, Wong SME, Peters MC, Mochly-Rosen D, Yeomans DC, Kendig JJ. Protein Kinase $C \mathcal{E}$ and $\gamma$ : Involvement in Formalin-Induced Nociception in Neonatal Rats. J Pharmacol and Exp Ther. 2004;309:616-25.

27. Taha R, Blaise GA. Update on the pathogenesis of complex regional pain syndrome: Role of oxidative stress. Can J Anesth/J Can Anesth. 2012;59:875-81.

28. Pinho-Ribeiro FA, Hohmann MS, Borghi SM, Zarpelon AC, Guazelli CF, Manchope MF, Casagrande R, Verri WA Jr. Protective effects of the flavonoid hesperidin methyl chalcone in inflammation and pain in mice: Role of TRPV1, oxidative stress, cytokines and NF-KB. Chem Biol Interact. 2015;228: $88-99$. 